\title{
Bacterias diazotróficas con actividad promotora del crecimiento vegetal en Daucus carota L.
}

\section{Diazotrophic bacteria with activity promoting vegetable growth in Daucus carota $L$.}

Fecha de recepción: 2 de septiembre de 2017

Fecha de aprobación: 5 de diciembre de 2017
Jennifer Gaviria-Giraldo ${ }^{1}$

Gloria María Restrepo-Franco ${ }^{2}$

Narmer Fernando Galeano-Vanegas ${ }^{3}$ Annia Hernández-Rodríguez ${ }^{4}$

DOI: https://doi.org/10.19053/01228420.v15.n1.2018.7753

\section{Resumen}

Este trabajo se propuso aislar bacterias diazotróficas con actividad promotora del crecimiento vegetal, asociadas al cultivo de zanahoria. Se realizaron 3 muestreos, a los 30, 60 y 115 días, en una granja ubicada en una zona rural del municipio de Manizales (Caldas). El aislamiento de las bacterias diazotróficas se efectuó en medios de cultivo semisólidos libres de nitrógeno. A los aislamientos obtenidos se les realizó descripción macro y microscópica, identificación bioquímica y molecular. Se evaluaron características como determinación de compuestos indólicos, actividad nitrogenasa y solubilización de fosfatos. Como cepa patrón se empleó Gluconacetobacter diazotrophicus ATCC 49037. Se recuperaron 20 aislamientos asociados a la rizosfera y 12 al rizoplano. La identificación molecular mostró cinco géneros presentes: Rhizobium, Achromobacter, Bacillus, Enterobacter y Stenotrophomonas. La producción de compuestos indólicos presentó concentraciones entre 9,73 y $112,8 \mu \mathrm{g} / \mathrm{mL}$. La cepa patrón presentó una actividad mayor, con una producción de compuestos indólicos de 172,5 $\mu \mathrm{g} / \mathrm{mL}$. En la actividad nitrogenasa los aislamientos GIBI411, 394 y 399 tuvieron una actividad mayor o similar a la cepa patrón. Los aislamientos más eficientes en la solubilización de fosfato tricálcico fueron GIBI378 y 385. La solubilización de fosfato de aluminio se valoró por el índice de producción de ácido, siendo los aislamientos GIBI378, 391, 387 y 388 los de mejor comportamiento en esta variable. Los aislamientos encontrados son candidatos potenciales para desarrollar nuevos procesos biotecnológicos para la producción de nuevos biofertilizantes alternativos, considerando las importantes propiedades de promoción del crecimiento vegetal determinadas en este trabajo.

Palabras clave: actividad nitrogenasa; fijación de nitrógeno; solubilización de fosfato; zanahoria.

1 M.Sc. Universidad Católica de Manizales (Manizales, Caldas). jgaviria@ucm.edu.co. ORCID: 0000-0002-4072-499X.

2 Ph.D. Universidad Católica de Manizales (Manizales, Caldas). grestrepo@ucm.edu.co. ORCID: 0000-0003-0443-0369.

3 Universidad Católica de Manizales (Manizales, Caldas).ngaleano@ucm.edu.co. ORCID: 0000-0002-8337-2824.

4 Ph.D. Universidad de la Habana (La Habana, Cuba). annia@fbio.uh.cu. ORCID: 0000-0002-0471-3089. 


\begin{abstract}
This study aimed at isolating diazotrophic bacteria with promoting activity of vegetal growth, associated to carrot culture. Samples were taken at 30,60 and 115 days in a farm located in a rural area of the municipality of Manizales (Caldas). The diazotrophic bacteria were isolated in semi-solid culture media free of nitrogen. We described the macro and microscopic features of the obtained isolations, and identified them biochemically and molecularly. In addition, we evaluated indole compounds, nitrogenase activity and solubilization of phosphates. Gluconacetobacter diazotrophicus ATCC 49037 was used as a standard strain. We recovered 20 isolates associated to rhizosphere and 12 to rhizoplane. The molecular identification showed the presence of five genera: Rhizobium, Achromobacter, Bacillus, Enterobacter, and Stenotrophomonas. The production of indole compounds presented concentrations between 9.73 and 112.8 $\mu \mathrm{g} / \mathrm{mL}$. The standard strain showed a higher activity, with an indolic compound production of $172.5 \mu \mathrm{g} /$ $\mathrm{mL}$. Regarding nitrogenase activity, the isolates GIBI411, 394 and 399 had an activity greater or similar to the standard strain. The most efficient isolates in the solubilization of tricalcium phosphate were GIBI378 and 385. The solubilization of aluminum phosphate was assessed by the acid production index, with the isolates GIBI378, 391, 387 and 388 presenting the best behavior. The isolates found are potential candidates for the development of novel biotechnological processes to produce new alternative biofertilizers, considering the significant plant-growth promotion properties determined in this work.
\end{abstract}

Keywords: carrot; nitrogen fixation; nitrogenase activity; phosphate solubilization.

\title{
Para citar este artículo:
}

Gaviria-Giraldo J., Restrepo-Franco GM., Galeano-Vanegas NF., Hernández-Rodríguez A. Bacterias diazotróficas con actividad promotora del crecimiento vegetal en Daucus carota L. Ciencia y Agricultura. 2018; 15(1): 19-27. 


\section{Introducción}

La evolución de los sistemas agroindustriales desencadenó que las actividades humanas incrementaran la contaminación ambiental, principalmente por la utilización de fertilizantes de síntesis química, empleados para mejorar la calidad del suelo. La utilización de fertilizantes como fuente de nitrógeno $(\mathrm{N})$ está alrededor del $80 \%$ (1), sin embargo, después de su aplicación existen pérdidas por procesos como la volatilización, la desnitrificación y el lavado, lo que hace que estos productos sean contaminantes ambientales (2).

Las sales orgánicas están constituidas por $\mathrm{N}$, representado en aminoácidos y proteínas, que son de vital importancia para el desarrollo de las plantas y sus procesos fisiológicos. Aunque los compuestos de $\mathrm{N}$ orgánico en las plantas solo constituyen el 2 $\%$ o menos, estas requieren alrededor de un $25 \%$ en la producción de energía para los procesos de germinación (brotes) y fructificación de las raíces (3). La zanahoria (Daucus carota) es un cultivo de gran importancia en la alimentación humana, por su alto contenido de vitaminas, fuente de $\alpha y$ $\beta$-caroteno, contenido de fibra y molibdeno, que es importante para el metabolismo de las grasas y carbohidratos (4). El cultivo de zanahoria tiene requerimientos de fertilización específicos; una cosecha de zanahoria de $24 \mathrm{t} /$ ha consume del suelo $32 \mathrm{~kg}$ de nitrógeno, $18 \mathrm{~kg}$ de fósforo y $100 \mathrm{~kg}$ de potasio. El aporte de nitrógeno en forma de nitrito acelera el cambio de coloración de amarillo a rojo, sin embargo, el exceso induce un desarrollo foliar excesivo, disminución de carotenos y aumento de nitratos (5).

Las Bacterias Promotoras del Crecimiento Vegetal (BPCV) están asociadas a muchas de las especies de plantas que están presentes en la mayoría de los ambientes (6). Se encuentran ampliamente representadas en diversidad de géneros microbianos, permitiendo aumentar la disponibilidad de nutrientes, transformarlos a formas asimilables por la planta y producir sustancias promotoras del crecimiento (fitohormonas) (7). Dentro de las BPCV se encuentran las bacterias diazotróficas, con capacidad para fijar el nitrógeno atmosférico. Por todos estos motivos y considerando la necesidad de disponer de insumos orgánicos menos contaminantes, más amigables con el planeta, eficientes, que no provengan de compuestos fósiles, para tener una agricultura sostenible, es necesario realizar investigación y desarrollo de nuevas tecnologías. En este caso, es importante el estudio de comunidades bacterianas diazotróficas con características promotoras de crecimiento vegetal para desarrollar propuestas de inoculantes microbianos provenientes de aislamientos nativos, que mejoren la producción de los cultivos en comparación con los fertilizantes de síntesis química.

\section{Materiales y métodos}

Se realizaron tres muestreos aleatorios en tiempos diferentes de un mismo cultivo de zanahoria: a los 30, 60 y 115 días. El cultivo de zanahoria estaba establecido en la Granja Tesorito, de la Universidad de Caldas, ubicada en el sector de 'Maltería' de la ciudad de Manizales, departamento de Caldas, a una Altitud de 2344 m s.n.m., Latitud $5^{\circ} 1,77$ Norte, Longitud $75^{\circ} 26,147$ Oeste. En cada tiempo de evaluación se recolectaron cuatro muestras de zanahoria, dos de cada surco seleccionado aleatoriamente; igualmente, se tomaron muestras de suelo de la rizosfera de cada sitio. Las muestras fueron almacenadas de manera individual en bolsas con cierre hermético.

El aislamiento de las bacterias diazotróficas se realizó a partir del rizoplano presente en la ectodermis de la zanahoria y del suelo de la rizosfera. El material vegetal se lavó con agua de la llave y, posteriormente, con agua destilada (8). Se realizaron cortes de la ectodermis, se pesaron y homogeneizaron, empleando un mortero estéril. Se utilizó un volumen de solución salina al 0,85\%, con Tween ${ }^{\circledR}$ 80 suficiente para obtener una dilución $1 / 10$, y se continuaron realizando diluciones seriadas hasta $10^{-5}$. Las muestras de suelo también fueron diluidas hasta $10^{-5}$, para su procesamiento. Los medios semisólidos libres en nitrógeno, NFB (9), JNFB (10) y LGI-P (8) fueron inoculados por triplicado con $0,1 \mathrm{~mL}$ de las diluciones comprendidas entre $10^{-2}$ y $10^{-5}(11)$. Los medios se llevaron a incubación a $30{ }^{\circ} \mathrm{C}$ (7-5 días). Se consideraron cultivos positivos 
para bacterias diazótrofas, aquellos con formación de una película superficial en el medio. Se realizó reinoculación de los cultivos positivos, en los mismos medios semisólidos del aislamiento primario. Posteriormente se realizó aislamiento de las bacterias asociadas a las películas recuperadas, en los medios de cultivo sólidos NFB, JNFB y LGI-P, los cuales se incubaron durante 5 días a $30{ }^{\circ} \mathrm{C}$. Las colonias recuperadas se sembraron nuevamente en los medios semisólidos, para verificar su comportamiento frente a la fijación biológica de nitrógeno.

A los aislamientos de bacterias diazotróficas recuperadas se les realizó descripción macroscópica en agar papa (9) y microscópica por medio de coloración de Gram. La identificación bioquímica se realizó por métodos convencionales e incluyó las pruebas de determinación de oxidasa, catalasa, reducción de nitratos, utilización de urea, hidrólisis de la gelatina y crecimiento en diferentes fuentes de carbono, como glucosa, sacarosa, inositol, sorbitol, arabinosa, galactosa y rafinosa (12).

Se realizó identificación molecular de los aislamientos obtenidos, a través de la extracción del ADN por medio del Kit PureLink Genomic DNA, recomendado para bacterias Gram negativas; luego, se realizó la cuantificación de ácidos nucleicos por espectrofotometría, utilizando el NanoDrop ${ }^{T M}$ 2000 a 260 nm, y se calculó la razón 260/280 y 260/230 en búsqueda de determinar la concentración y calidad del ADN, respectivamente. Para continuar con el proceso se realizó la amplificación del ADNr de la subunidad 16S, para lo cual se utilizaron cebadores universales RB; este procedimiento se llevó a cabo en un termociclador MS mini bajo los siguientes parámetros de amplificación: desnaturalización inicial $95{ }^{\circ} \mathrm{C}$ por 10 minutos, desnaturalización adicional por 1 minuto a $92{ }^{\circ} \mathrm{C}$, 35 ciclos de $72{ }^{\circ} \mathrm{C}$ cada uno durante 2 minutos. La amplificación por PCR del ADN se verificó mediante electroforesis en gel de agarosa al $1 \%$, utilizando como buffer de carga EZ-VISION ONE. La secuenciación de los productos de la PCR se realizó en la compañía Macrogen ${ }^{\circledR}$ (República de Corea). Se realizó un análisis de calidad de las secuencias obtenidas mediante el programa CLC
MainWorkbench (Qiagen Company, EUA), usando como criterio de aceptación un valor Phred mayor a 30 por base. Las secuencias fueron comparadas con la base de datos $n t$ del NCBI, utilizando el programa BLASTN ver. 2.2.27+ (13). Se consideraron como un acierto (hit) los alineamientos con un valor-e inferior a $1 \times 10^{-5}$, con una similitud y cobertura superior al $80 \%$ de la región amplificada con los primers que amplifican la región inicial del gen $16 \mathrm{~S}$ (800 pb). El resultado fue validado usando BLASTN ver. 2.2.27+, contra la base de datos taxonómica RDP (Ribosomal Database Project; RDP Release 11, Update 3: September 17, 2014).

La actividad nitrogenasa de las bacterias fue determinada en el medio de cultivo correspondiente al del aislamiento primario (NFB, JNFB o LGI-P) sin indicador de $\mathrm{pH}$, mediante el método de Reducción del Acetileno (ARA) (14-16), con algunas modificaciones. Una suspensión de $20 \mu \mathrm{L}$, a una concentración de $10^{8}$ células/Ml, fue inoculada en $5 \mathrm{~mL}$ de medio LGI-P, los cuales fueron incubados a $30{ }^{\circ} \mathrm{C}$ durante $48 \mathrm{~h}$. Posteriormente, se sustituyó el $10 \% \mathrm{v} / \mathrm{v}$ de la atmósfera del vial de cultivo con el gas acetileno. Luego de la incubación durante $24 \mathrm{~h}$ a $30{ }^{\circ} \mathrm{C}$, se analizaron muestras de gas de 500 $\mu \mathrm{L}$ en un cromatógrafo de gases-masas acoplado con un detector de ioinización de llama GCMSQP2010 PLUS (Shimadzu ${ }^{\circledR}$ ), usando una columna GS-Alumina (Agilent Technologies ${ }^{\circledR}$ ). Cada muestra se evaluó seis veces. Los resultados obtenidos se expresaron en $\mathrm{nmol}$ de acetileno reducido/( $\mathrm{mL} \times \mathrm{h})$ y en nmol de acetileno reducido/ $\left(10^{8}\right.$ células $\left.x h\right)$.

Los compuestos indólicos totales se determinaron mediante espectofotometría, empleando el reactivo de Salkowski (17-19). Se partió de una colonia recuperada en medio Agar Papa Dextrosa, la cual se pasó a un medio DYGS y se incubó en agitación a $30{ }^{\circ} \mathrm{C}$, a $150 \mathrm{rpm}$, entre 24 y 48 horas. Se realizaron dos lavados con agua destilada estéril y se preparó el inóculo con una concentración de $10^{8}$ células/mL. La producción de compuestos indólicos se indujo mediante la adición de L-triptófano $(1,01$ \%) (Panreac ${ }^{\circledR}$, EUA) en medio DYGS, a $30{ }^{\circ} \mathrm{C}$ y 150 rpm durante 60 horas. La determinación de compuestos indólicos se realizó en el sobrenadante mediante reacción con reactivo de Salkowski y 
lectura de absorbancia a $540 \mathrm{~nm}$. Cada muestra se procesó por triplicado. Los niveles de compuestos indólicos se estimaron a partir de una curva patrón de 150 ppm de ácido indolacético (MERCK ${ }^{\circledR}$, EUA) y se expresaron en $\mu \mathrm{g} / \mathrm{mL}$.

La solubilización de fósforo se valoró empleando el medio NBRIP con verde de bromocresol (22 $\mathrm{mg} / \mathrm{L})(20)$, el cual fue suplementado individualmente con $5 \mathrm{~g} / \mathrm{L}$ de $\mathrm{Ca}_{3}\left(\mathrm{PO}_{4}\right)_{2}$ o AlPO . En cada placa de medio se depositó un volumen de $20 \mu \mathrm{L}$, por triplicado, proveniente del inóculo. Se realizaron lecturas a las 24, 48 y 72 horas, en las cuales se determinó el diámetro de la colonia y el diámetro de los halos de solubilización, con el fin de calcular el índice de solubilización (21) y el porcentaje de eficiencia de solubilización (22). Adicionalmente, se evaluó la producción de ácidos orgánicos mediante el viraje del indicador de $\mathrm{pH}$ del medio de cultivo.

Con los resultados obtenidos se realizó análisis de varianza en el programa $R$, versión 3.4.0, paquete agricolae versión 1.2-8. Se hizo comparación de medias a través de la prueba de partición de promedios o test de Duncan (valor-p $<0,05$ ).

\section{Resultados y discusión}

Se obtuvieron 32 aislamientos de bacterias diazotróficas, de los cuales, el 18,75 \% mostraron, en la coloración de Gram, bacilos Gram positivos, y el $81,25 \%$, bacilos Gram negativos. De acuerdo con las características macro- y microscópicas evidenciadas, se identificaron 6 morfotipos en las bacterias aisladas. El morfotipo 1 presentó colonias cremosas, brillantes, mucoides, de bordes irregulares en Agar Papa Dextrosa (APD), y en el análisis microscópico se observaron bacilos Gram negativos (100x). En el morfotipo 2 se observaron bacilos Gram negativos con características macroscópicas de colonias brillantes, color crema, con bordes regulares. El morfotipo 3 presentó colonias secas, opacas, de bordes irregulares en agar APD y bacilos Gram positivos en la coloración de Gram. El morfotipo 4 presentó bacilos Gram negativos y colonias mucoides, blancas con bordes irregulares. El morfotipo 5 está representado por bacilos cortos Gram negativos con colonias cremosas, brillantes, convexas, con bordes regulares en agar APD. Posteriormente, se realizaron pruebas bioquímicas a los aislados, donde se determinó la producción de enzimas, dando como resultado que el $100 \%$ de los aislados producen la enzima catalasa; el $97 \%$, la enzima ureasa; el $44 \%$, la enzima oxidasa, y ninguno de los aislados produjeron enzimas del tipo proteasas. Las fuentes de carbono más utilizadas por los aislados correspondieron a citrato, glucosa, rafinosa y arabinosa, con un $75 \%, 44 \%$, $44 \%$ y $41 \%$, respectivamente.

De los 32 aislamientos de bacterias diazotróficas obtenidos, 20 provenían de rizosfera y 12 de rizoplano. El 81,25 \% de los aislamientos fueron recuperados en el primer muestreo, correspondiente a los 30 días de establecimiento del cultivo. Lo anterior se puede explicar debido a que en los primeros días del cultivo ocurren estadios como la germinación, desarrollo de las hojas, formación del tallo principal y despliegue completo de cotiledones, en los cuales hay una alta actividad microbiana en la rizosfera (23), la cual se pudo expresar en el primer tiempo de evaluación a través de los aislamientos obtenidos.

En la identificación molecular de los 32 aislados se obtuvieron como resultado cinco géneros bacterianos, siendo Stenotrophomonas sp. el de mayor representación, con el 37,5\%, seguido de géneros como Bacillus, Achromobacter y Rhizobium, cada uno con el 18,8 \% de representación. El género de menor representación fue Enterobacter, con un acierto del 6,25\%. Aunque han sido poco estudiadas las poblaciones bacterianas diazotróficas presentes en el cultivo de zanahoria, se han realizado estudios en los cuales se han encontrado diferentes géneros de bacterias asociados al cultivo, como Pseudomonas spp., Agrobacterium spp., Pantoea spp., Enterobacter spp., Bacillus spp., Klebsiella spp., Stenotrophomonas spp. (24) y Gluconacetobacter diazotrophicus (25). Los géneros Bacillus spp., Achromobacter spp. y Enterobacter spp. se han descrito como promotores de crecimiento vegetal en especies forestales alto-andinas colombianas (26), y los géneros Achromobacter spp. y Enterobacter spp., como solubilizadores de fosfato y fijadores de nitrógeno. El género Stenotropho- 
monas spp. también ha sido aislado e identificado a partir de cultivos de caña de azúcar (27).

Los valores de ARA en los aislamientos evaluados oscilaron entre 0,0073 y $9,2940 \%$. La mejor actividad la presentó el aislamiento GIBI411, correspondiente a $B$. weihenstephanensis, el cual tuvo un porcentaje mayor de reducción del acetileno $(9,294 \%)$ que la cepa patrón $(5,807 \%)$, con diferencias estadísticas significativas. Los aislamientos GIBI394 y 399 tuvieron un rendimiento estadísticamente similar al obtenido con la cepa ATCC 49073 (Tabla I). La producción de compuestos indólicos por los aislamientos evaluados presentaron diferentes concentraciones, que van desde 9,73 hasta $172,5 \mu \mathrm{g} / \mathrm{mL}$. La cepa patrón tuvo el mejor rendimiento en la producción de compuestos indólicos $(172,50 \mathrm{ug} / \mathrm{mL})$, seguida por los aislamientos GIBI381 y 402, con diferencias estadísticas significativas, y un promedio de $60 \mu \mathrm{g} / \mathrm{mL}$ por debajo de los valores obtenidos con la cepa patrón (Tabla I).

El género Rhizobium spp. predominó en las muestras provenientes del rizoplano, lo que concuerda con los aislamientos realizados de este microorganismo a partir de muestras de rizosfera proveniente de diferentes plantas, como la canola (28); igualmente, este género bacteriano ha sido recuperado de nódulos de arbustos, cuyos aislamientos han presentado una producción de ácido 3-indolacético de $99,7 \mu \mathrm{g} / \mathrm{mL}$ (29), que es un valor contrastante en comparación con el de los 6 aislamientos obtenidos en este estudio (GIBI391, 385, 383, 388, 375 y 406), con resultados de 70,22, 69,43, 46,89, $43,24,41,34$ y $27,36 \mu \mathrm{g} / \mathrm{Ml}$, respectivamente. Bacillus spp. es un género ampliamente reportado como promotor del crecimiento vegetal (30); entre los aislamientos recuperados de este género, el mayor rendimiento en la producción de compuestos indólicos correspondió al GIBI410, con 68,88 $\mu \mathrm{g} / \mathrm{Ml}$, y el menor, al GIBI376, con una concentración de $11,72 \mu \mathrm{g} / \mathrm{mL}$, con diferencias estadísticas significativas entre ellos. Este resultado contrasta con lo reportado por Angulo et al. (31), quienes caracterizaron rizobacterias promotoras del crecimiento vegetal en plántulas de Eucalyptus, obteniendo aislamientos de $B$. weihenstephanensis que demostraron una producción de compuestos indólicos entre 5,38 y $6,35 \mu \mathrm{g} / \mathrm{mL}$.

Los aislamientos del género Enterobacter spp. (GIBI381 y 413) tuvieron una producción de compuestos indólicos de 112,80 y $74,80 \mu \mathrm{g} / \mathrm{Ml}$, respectivamente, valores cercanos a los obtenidos en el estudio realizado por Attar et al. (32), con una producción de $80 \mu \mathrm{g} / \mathrm{Ml}$; este género también ha sido aislado de caña de azúcar (27) y de cultivos de maíz, donde la producción de compuestos indólicos fue de 26,94 $\mu \mathrm{g} / \mathrm{mL}$ (33).

Finalmente, a los aislamientos recuperados se les determinó la capacidad de solubilización de fosfato en medio NBRIP con fosfato tricálcico y fosfato de aluminio. El aislamiento GIBI378, correspondiente a $B$. aerophilus, presentó los mayores valores en el IS en fosfato tricálcico y en el IPA en fosfato de aluminio $(3,037$ y 11,250$)$, con diferencias estadísticas significativas con respecto a la cepa patrón ATCC 49037, que presentó un IS en fosfato tricálcico de 2,727 y un IPA 10,139 en fosfato de aluminio (Tabla I). Es importante realizar pruebas de laboratorio con estos aislamientos en medio líquido y con el empleo de otras fuentes de fósforo, antes de realizar evaluaciones en campo y realizar la caracterización de los ácidos implicados en la solubilización $(34,35)$. 
Tabla I. Caracterización fisiológica de los aislados.

\begin{tabular}{|c|c|c|c|c|c|c|}
\hline \multirow{3}{*}{ Bacterias } & \multirow{3}{*}{ ARA $(\%)$} & \multirow{3}{*}{$\begin{array}{c}\text { AIA (ug/ } \\
m L)\end{array}$} & \multicolumn{4}{|c|}{ Solubilización de fosfatos } \\
\hline & & & \multicolumn{2}{|c|}{ Fosfato tricálcico } & \multicolumn{2}{|c|}{ Fosfato de aluminio } \\
\hline & & & IS (mm) & ES (\%) & IS (mm) & IPA $(\mathrm{mm})$ \\
\hline Rhizobium sp. GIBI375 & $0,0093^{\mathrm{e}}$ & $41,34^{\mathrm{pq}}$ & $1,000^{\mathrm{Imn}}$ & $0,000^{\ln }$ & $0,000^{c}$ & $0,000^{\mathrm{g}}$ \\
\hline Bacillus weihenstephanensis GIBI376 & $0,0086^{\mathrm{e}}$ & $11,72^{\text {st }}$ & $1,000^{\operatorname{lmn}}$ & $0,000^{\ln }$ & $0,000^{c}$ & $0,000^{\mathrm{g}}$ \\
\hline Bacillus aerophilus GIBI378 & $0,0092^{\mathrm{e}}$ & $49,48^{\text {no }}$ & $3,037^{\mathrm{a}}$ & $203,704^{a}$ & $1,000^{b}$ & $11,250^{\mathrm{a}}$ \\
\hline Stenotrophomonas sp. GIBI379 & $0,0086^{\mathrm{e}}$ & $59,20^{\mathrm{Im}}$ & $1,000^{\operatorname{lmn}}$ & $0,000^{\ln }$ & $1,000^{b}$ & $1,000^{f}$ \\
\hline Stenotrophomonas sp. GIBI380 & $0,0101^{\mathrm{e}}$ & $72,16^{\text {ghij }}$ & $2,121^{\mathrm{de}}$ & $112,099^{\text {de }}$ & $1,000^{\mathrm{b}}$ & $9,694^{c}$ \\
\hline Enterobacter sp. GIBI381 & $0,0076^{\mathrm{e}}$ & $112,80^{\mathrm{b}}$ & $1,208^{\mathrm{klm}}$ & $20,811^{\mathrm{klm}}$ & $0,000^{c}$ & $0,000^{\mathrm{g}}$ \\
\hline Stenotrophomonas sp. GIBI382 & $0,0074^{\mathrm{e}}$ & $88,04^{\text {de }}$ & $2,611^{\mathrm{c}}$ & $161,111^{\mathrm{c}}$ & $1,000^{b}$ & $10,139^{b}$ \\
\hline Rhizobium sp. GIBI383 & $0,0078^{\mathrm{e}}$ & $46,89^{\text {op }}$ & $1,777^{\text {fgh }}$ & $77,725^{\mathrm{fg}}$ & $1,000^{b}$ & $5,736^{d}$ \\
\hline Stenotrophomonas sp. GIBI384 & $0,0077^{\mathrm{e}}$ & $54,53^{\mathrm{mn}}$ & $1,536^{\mathrm{ij}}$ & $53,571^{\mathrm{ij}}$ & $1,000^{\mathrm{b}}$ & $1,737^{\mathrm{e}}$ \\
\hline Rhizobium sp. GIBI385 & $0,0078^{\mathrm{e}}$ & $69,43^{\text {hijk }}$ & $2,920^{\mathrm{ab}}$ & $197,975^{\mathrm{ab}}$ & $0,000^{c}$ & $0,000^{\mathrm{g}}$ \\
\hline Achromobacter spanius GIBI387 & $0,0076^{\mathrm{e}}$ & $40,14^{\mathrm{q}}$ & $1,833^{\mathrm{fg}}$ & $83,333^{\mathrm{fg}}$ & $1,014^{a}$ & $11,111^{\mathrm{a}}$ \\
\hline Rhizobi & 0,00 & $43,24^{\text {opq }}$ & $1,679^{\text {ghi }}$ & $67,901^{\text {ghi }}$ & $1,000^{\mathrm{b}}$ & $111^{\mathrm{a}}$ \\
\hline Stenotrophomonas sp. GIBI389 & $0,0076^{e}$ & $30,32^{r}$ & $1,769^{\mathrm{gh}}$ & $76,852^{\text {gh }}$ & $1,000^{b}$ & \\
\hline Stenotrophomonas maltophilia GIB & 0,00 & 30,4 & $0,000^{0}$ & $0,000^{\ln }$ & $0,000^{c}$ & \\
\hline Rhizobium sp. GIBI391 & $0,0085^{\mathrm{e}}$ & $70,22^{\text {hijk }}$ & $1,828^{\mathrm{fg}}$ & $82,840^{\mathrm{fg}}$ & $1,000^{b}$ & $11,250^{\mathrm{a}}$ \\
\hline Achromobacter spanius GI & $0,0085^{\mathrm{e}}$ & $78,08^{\mathrm{fg}}$ & $1,997^{\text {ef }}$ & $99,683^{\text {ef }}$ & $1,000^{\mathrm{b}}$ & $9,889^{\mathrm{bc}}$ \\
\hline Achromobacter spanius GI & $6,0560^{\mathrm{b}}$ & $75,86^{\text {fgh }}$ & $2,267^{\mathrm{d}}$ & $126,698^{\mathrm{d}}$ & $1,000^{\mathrm{b}}$ & $10,000^{\mathrm{bc}}$ \\
\hline Achromobacter spanius GIBI397 & $0,0073^{\mathrm{e}}$ & $56,94^{\mathrm{lm}}$ & $1,022^{\operatorname{lmn}}$ & $2,222^{\operatorname{lmn}}$ & $0,000^{c}$ & $0,000^{\mathrm{g}}$ \\
\hline Achromobacter xylosoxidans GIBI398 & $0,0080^{\mathrm{e}}$ & $63,60^{k 1}$ & $0,000^{0}$ & $0,000^{\ln }$ & & \\
\hline Stenotrophomonas maltophilia GIBI399 & 5,31 & $70,08^{\text {hijk }}$ & $1,000^{\operatorname{Imn}}$ & $0,000^{\ln }$ & $0,000^{c}$ & \\
\hline Stenotrophomonas maltophilia GIBI400 & $0,0081^{\mathrm{e}}$ & $17,46^{\mathrm{s}}$ & $0,000^{0}$ & $0,000^{\ln }$ & $0,000^{c}$ & $0,000^{\mathrm{g}}$ \\
\hline Stenotrophomonas sp. GIBI401 & $0,0087^{\mathrm{e}}$ & $39,67^{q}$ & $2,172^{\mathrm{de}}$ & $117,196^{\text {de }}$ & $1,000^{b}$ & $10,000^{\mathrm{bc}}$ \\
\hline Stenotrophomonas rhizophila GIBI402 & & $101,60^{\mathrm{c}}$ & $1,397^{\mathrm{jk}}$ & $39,735^{\mathrm{jk}}$ & $1,000^{b}$ & \\
\hline Stenotrophomonas sp. GIBI405 & $0,0076^{\mathrm{e}}$ & $66,10^{\mathrm{jk}}$ & $0,000^{\circ}$ & $0,000^{\ln }$ & $0,000^{c}$ & $0,000^{\mathrm{g}}$ \\
\hline Rhizobium sp. GIBI406 & $0,0083^{\mathrm{e}}$ & $27,36^{r}$ & $0,000^{0}$ & $0,000^{\ln }$ & $0,000^{c}$ & $0,000^{\mathrm{g}}$ \\
\hline Bacillus weihenstephanensis GIBI407 & $0,0092^{\mathrm{e}}$ & $16,63^{\mathrm{s}}$ & $1,000^{\operatorname{lmn}}$ & $0,000^{\ln }$ & $0,000^{c}$ & $0,000^{\mathrm{g}}$ \\
\hline Achromobacter spanius GIBI409 & $0,0085^{\mathrm{e}}$ & $81,37^{\text {ef }}$ & $1,467^{\mathrm{ij}}$ & $46,667^{\mathrm{ij}}$ & $0,000^{c}$ & $0,000^{\mathrm{g}}$ \\
\hline Bacillus weihenstephanensis GIBI410 & $0,0078^{\mathrm{e}}$ & $68,88^{\mathrm{ijk}}$ & $0,889^{n}$ & $0,000^{\ln }$ & $0,000^{c}$ & $0,000^{g}$ \\
\hline Bacillus weihenstephanensis GIBI411 & $9,2940^{\mathrm{a}^{* *}}$ & $9,73^{t}$ & $1,222^{k \mathrm{k}}$ & $22,222^{\mathrm{kl}}$ & $0,000^{c}$ & $0,000^{\mathrm{g}}$ \\
\hline Bacillus weihenstephanensis GIBI412 & $0,0086^{\mathrm{e}}$ & $25,65^{r}$ & $1,641^{\text {ghi }}$ & $64,074^{\text {ghi }}$ & $0,000^{c}$ & $0,000^{g}$ \\
\hline Enterobacter sp. GIBI413 & $1,6620^{\mathrm{d}}$ & $74,80^{\text {fghi }}$ & $1,539^{\text {hij }}$ & $53,872^{\text {hij }}$ & $1,000^{b}$ & $5,540^{\mathrm{d}}$ \\
\hline Stenotrophomonas maltophilia GIBI414 & $0,0082^{\mathrm{e}}$ & $93,26^{\mathrm{d}}$ & $1,501^{\mathrm{ij}}$ & $50,117^{\mathrm{ij}}$ & $1,000^{\mathrm{b}}$ & $10,139^{b}$ \\
\hline ATCC49037 & $5,8070^{\mathrm{b}}$ & $172,5^{\mathrm{a}^{* *}}$ & $2,727^{\mathrm{bc}}$ & $172,745^{\mathrm{bc}}$ & $1,000^{\mathrm{b}}$ & $10,139^{b}$ \\
\hline
\end{tabular}

Letras no comunes en las columnas indica diferencias significativas según prueba de

Duncan para $\mathrm{p}<0,05$

\section{Conclusiones}

Se obtuvieron aislamientos de bacterias diazotróficas con potencial para la formulación de inoculantes microbianos para el cultivo de zanahoria, con diferentes características de promoción del crecimiento vegetal. Los mejores resultados in vitro se alcanzaron con los aislamientos GIBI411 (B. weihenstephanensis), en la actividad de reducción del acetileno, la cual está relacionada con la actividad nitrogenasa para la fijación biológica de nitrógeno; GIBI381, de Enterobacter sp., en la producción de compuestos indólicos, y, finalmente, el GIBI378, de $B$. aerophilus, en la solubilización de fosfato tri- 
cálcico y de aluminio. Es importante realizar pruebas en campo con estos aislamientos, con el fin de validar su comportamiento in vivo.

\section{Referencias}

(1) Domínguez A., Prieto RG., Achkar M. Perfil ambiental del Uruguay: 2000. 2007; 22: 35-36. Montevideo: Ecoteca Series.

(2) Stein L., Klotz M. The nitrogen cycle. Curr Biol, 2016; 26: R94-R98. DOI: http://doi.org/10.1016/j.cub.2015.12.021.

(3) Bloom A. The increasing importance of distinguishing among plant nitrogen sources. Curr Opin Plant Biol. 2015; 25: 10-16. DOI: http://doi.org/10.1016/j.pbi.2015.03.002.

(4) Da Silva Dia JC. Nutritional and health benefits os carrots and their seed extracts. Food Nutr Sci. 2014; 5: 2147-2156. DOI: http://doi.org/10.4236/fns.2014.522227.

(5) Reina CE., Bonilla JF. Manejo postcosecha y evaluación de calidad para zanahoria (Daucus carota L.) que se comercializa en la ciudad de Neiva. Facultad de Ingeniería, Programa de Ingeniería Agrícola, 1997.

(6) Compant S., Reiter B., Sessitsch A., Nowak J., Clément C., Barka E. Endophytic colonization of Vitis vinifera L. by plant growth-promoting bacterium Burkholderia sp. Appl Enviro Microbiol. 2005; 71(4): 1685-1693. DOI: http://doi.org/10.1128/ AEM.71.4.1685-1693.2005.

(7) Beneduzi A., Moreira F., Costa PB., Vargas LK., Lisboa BB., Favreto R., Baldani JI., Passaglia LMP. Diversity and growth promoting evaluation abilities of bacteria isolated from sugarcane cultivated in the South of Brazil. Appl Soil Ecol. 2013; 63: 94 104. DOI: http://doi.org/10.1016/j.apsoil.2012.08.010.

(8) Cavalcante VA., Döbereiner J. A new acid -tolerant nitrogenfixing bacterium associated with sugarcane. Plant Soil. 1988; 108: 23-31. DOI: http://doi.org/10.1007/BF02370096.

(9) Baldani VLD., Döbereiner J. Host plant specificity in the infection of cereal with Azospirillum spp. Soil Biol Biochem. 1980; 12: 433-439. DOI: http://doi.org/10.1016/0038-0717(80)900218.

(10) Baldaniet AL. Meios de cultura específicos para o isolamento de bactérias enfofiticas que fixam $\mathrm{N}_{2}$. Comunicado técnico. CNPAB, 1996; 12:3.

(11) Döbereiner J. Forrage grasses and grain crops. En: Methods for evaluating biological nitrogen fixation, 1980.

(12) Dong Z., Heydrich M., Bernard K., McCully ME. Further evidence that the $\mathrm{N}_{2}$-fixing endophytic bacterium from the intercellular spaces of sugarcane stems is Acetobacter diazotrophicus. Appl Environ Microbiol. 1995; 61(5): 1843-1846.

(13) Altschul SF., Gish W., Miller W., Myers EW., Lipman DJ. Basic local alignment search tool. J Mol Biol. 1990; (215): 403-410. DOI: http://doi.org/10.1016/S0022-2836(05)80360-2.

(14) Muthukumarasamy R., Rebathi G., Lakshminarasimhan C. Influence of $\mathrm{N}$ fertilization on the isolation of Acetobacter diazotrophicus and Herbaspirillum spp. from Indian sugarcane varieties. Biol Fertil Soils. 1999; 29: 157-164. DOI: http://doi. org/10.1007/s003740050539.
(15) Videira S., Simões JL., Baldani V. Metodologia para isolamento e posicionamento taxonômico de bactérias diazotróficas oriundas de plantas não-leguminosas. Agrobiología. 2007; 234: 74.

(16) Boddey LH., Boddey RM., Rodríguez BJ., Urquiaga S. A avaliação da fixação biológica de $\mathrm{N}_{2}$ associada a leguminosas e nãoleguminosas utilizando a técnica da redução do acetileno: histórica, teoria e prática. Agrobiología. 2007; 245: 43.

(17) Gordon S., Werber R. Colorimetric estimation of indole acetic acid. Plant Physiol. 1950; 26: 192-195. DOI: http://doi. org/10.1104/pp.26.1.192.

(18) Glickmann E., Dessaux Y. A critical examination of the specificity of the Salkowski reagent for indolic compounds produced by phytopathogenic bacteria. Appl Environ Microbiol. 1995; 61(2): 793-796.

(19) Sarwar M., Kremer RJ. Determination of bacterially derived auxins using a microplate method. Lett Appl Microbiol. 1995; 20: 282-285. DOI: http://doi.org/10.1111/j.1472-765X.1995. tb00446.x.

(20) Nautiyal CS. An efficent microbial growth medium for screening phosphate solubilizing microorganisms. FEMS Microbiol Lett. 1999; 170: 265-270. DOI: http://doi. org/10.1111/j.1574-6968.1999.tb13383.x.

(21) Kumar V., Narula N. Solubilization of inorganic phosphates and growth emergence of wheat as affected by Azotobacter chromococcum mutants. Biol Microbiol. 1999; 29: 301-305.

(22) Nguyen CW., Yan WF., Tacon L., Lapayrie F. Genetic variability of phosphate solubilizing activity by monocaryotic and dicaryotic mycelia of the ectomycorrhizal fungus Laccaria bicolor (Maire) P.D. Orton. Plant Soil. 1992; 143: 193-199. DOI: http:// doi.org/10.1007/BF00007873.

(23) Feller C., Bleiholder H., Buhr L., Hack H., Hess M., Klose R., Meier U., Stauss R., Van T. Phänologische entwicklungsstadien von gemüsepflanzen: I. Zwiebel, wurzel, knollen und blattgemüse codierung und beschreinbung nach der erweiterten $\mathrm{BBCH}$-skala mit abbildungen. Nachrichtenbl. Deut. Pflanzenschtzd. 1995; 47: 193-206.

(24) Surette MA., Sturz AV., Lada RR., Nowak J. Bacterial endophytes in processing carrots (Daucus carota L. var. sativus): their localization, population density, biodiversity and their effects on plant growth. Plant Soil. 2003; 254: 381-390. DOI: http://doi. org/10.1023/A:1024835208421.

(25) Madhaiyan M., Saravanan VS., Bhakiya D., Hyoungseok L., Thenmozhi R., Hari K., Sa. T. Occurrence of Gluconacetobacter diazotrophicus in tropical and subtropical plants of Western Ghats, India. Microbiol Res. 2004; 159: 233-243. DOI: http:// doi.org/10.1016/j.micres.2004.04.001.

(26) Martínez NP., García G. Bacterias diazotróficas y solubilizadoras de fósforo aisladas de las especies forestales altoandinas colombianas Weinmannia tomentosa y Escallonia myrtilloides. Intropica. 2010; 5: 63-76.

(27) Taulé C., Mareque C., Barlocco C., Hackembruch F., Sicardi M., Battistoni F. Bacterias promotoras del crecimiento vegetal asociadas a caña de azúcar. Serie FPTA-INIA. 2014; 54: 9-46. 
(28) Farina R., Beneduzi A., Ambrosini A., De Campos SB., Lisboa BB., Wendisch V., Vargas LK., Passaglia LMP. Diversity of plant growth-promoting rhizobacteria communities associated with the stage of canola growth. App/ Soil Ecol. 2012; 55: 44-52. DOI: http://doi.org/10.1016/j.apsoil.2011.12.011.

(29) Datta C., Basu PS. Indole acetic acid production by Rhizobium species from root nodules of a leguminous shrub, Cajanus cajan. Microbiol Res. 2000; 155(2): 123-127. DOI: http://doi. org/10.1016/S0944-5013(00)80047-6.

(30) Idris ESE., Iglesias DJ., Talon M., Borriss R. Tryptophan-dependent production of indole-3-acetic acid (IAA) affects level of plant growth promotion by Bacillus amyloliquefaciens FZb42. Am Phytoplathol Soc. 2007; 20(6): 619-626.

(31) Angulo VC., Sanfuentes EA., Rodríguez F., Sossa KE. Caracterización de rizobacterias promotoras de crecimiento en plántulas de Eucalyptus nitens. Rev Argent Microbiol. 2014; 46(4): 338-347. DOI: http://doi.org/10.1016/S0325-7541(14)70093-8.

(32) Attar YC., Mali SD., Kamble PP. Study of phosphate solubilising Enterobacter cloacae sub sp. cloacae strain YCA for production of plant growth promoting substances. Int I Pure Appl Biosci. 2015; 3(1): 71-80.

(33) Montañez A., Rodríguez BA., Barlocco C., Beracochea M. Characterization of cultivable putative endophytic plant growth promoting bacteria associated with maize cultivars (Zea mays L.) and their inoculation effects in vitro. Appl Soil Ecol. 2012; 58: 21-28. DOI: http://doi.org/10.1016/j.apsoil.2012.02.009.

(34) Bashan Y., Kamnev AA., Bashan LE. Tricalcium phosphate is inappropriate as a universal selection factor for isolating and testing phosphate-solubilizing bacteria that enhance plant growth: a proposal for an alternative procedure. Biol Fertil Soils. 2013; 49: 465-479. DOI: http://doi.org/10.1007/s00374-012-0737-7.

(35) Restrepo Franco GM., Marulanda Moreno S., de la Fe Pérez Y., Díaz de la Osa A., Baldani VL., Hernández-Rodríguez A. Bacterias solubilizadoras de fosfato y sus potencialidades de uso en la promoción del crecimiento de cultivos de importancia económica. Rev CENIC Ciencias Biol. 2015; 46(1): 63-76. 
